\title{
Snap-through Buckling of Shallow Spherical Shells under Ring Loads
}

\author{
Esra Eylem KARATAŞ ${ }^{1}$ \\ Receb Faruk YÜKSELER ${ }^{2}$
}

\begin{abstract}
Snap-through buckling behaviour of rigid vinyl polyethylene shallow spherical shells, undergoing large displacements, subjected to static ring loads is investigated by using finite difference and the Newton-Raphson Method. The load-deflection diagrams corresponding to various values of thickness, depth and ring diameter of the shell with simply supported and clamped edges are sketched and compared. The accuracy of the used algorithm and the prepared computer program are tested by comparing some of the numerical results obtained in this study with those obtained by an experimental study, available in the relevant literature. Further steps on the concerning subject are achieved.
\end{abstract}

Keywords: Shallow spherical shell, nonlinear behaviour, large deflection, snap-through buckling, upper-limit point, rigid vinyl polyethylene, stability.

\section{INTRODUCTION}

For determining the load carrying capacities of structures the stability criterion, as well as the strength and stiffness criteria, must be considered especially for structures having thin-walled members. There are, mainly, two types instability: namely, bifurcation of equilibrium [1-11], and snap-buckling. Snap-through behaviour of structural elements (especially; shallow spherical shells, shallow arches and initially imperfect rods which have been used in many engineering, biological, industrial and scientific systems and have been subjected to severe external effects) is a very important physical phenomenon, not only for engineers but also for the scientists dealing with various structural systems. Although the snap-through behaviour of the relevant $\mathrm{c}$ structural elements resemble each other generally, the details of the concerning phenomenon (which is different for different structural elements, dimensional characteristics, materials used) can be crucially important from the point of design and

\section{Note:}

- This paper has been received on May 14, 2019 and accepted for publication by the Editorial Board on December 4, 2019.

- Discussions on this paper will be accepted by May 31, 2021.

- https://doi.org/10.18400/tekderg.565095

1 Yildiz Technical University, Department of Civil Engineering, İstanbul, Turkey - ekaratas@yildiz.edu.trhttps://orcid.org/0000-0003-1396-2463

2 Yildiz Technical University, Department of Civil Engineering, İstanbul, Turkey yukselerfaruk@gmail.com - https://orcid.org/0000-0002-2733-580X 
analysis of such systems. Therefore, it is not a surprise to observe publications on this subject receiving much attention for many years, including those in recent years [12-28].

Rigid vinyl polyethylene is a special material having appealing mechanical properties [https://www.aeplastics.com/rpvc/] used in many engineering systems (e.g. doorways, windows, waste water pipes, home plumbing pipes, profiles, extruded wire coverings, etc.). It was chosen to be used in the experimental study of Evan-Iwanowski et al [29] on the snapthrough behaviour of shallow, clamped, simply-supported, and free edge spherical shells and arches subjected to the ring loads and concentrated loads for various values of thickness, ring diameter, depth and other dimensions related with the details of their experiments. They concluded, importantly, that there was no snap-buckling for a clamped shallow spherical shell, subjected to the ring loads, having a ring diameter below a certain value depending on the dimensions of the shell. They made a similar comment for the simply-supported spherical shells, disregarding the second summit in their load-deflection diagrams. No comment was made, in their very rigorous study, about the probability of snap-buckling free situation via the appropriate choice of the thickness higher than a certain value depending on the chosen values of the ring diameter and dimensions of the shell. To the best of the authors' knowledge of the current study, there has been no other experimental study performed on this subject, using the same material.

Second study on the snap-through behaviour of spherical shells, using the same material, under the ring loads belonged to Cagan and Taber [30]. They considered deep as well as the shallow spherical shells with clamped edges only, and used a Newton-Raphson technique on discretized nonlinear shell equations. Comparing their numerical results with those of the experimental results of Evan-Iwanowski et al [29], they achieved good agreement generally. The results, they obtained, showed that (i) if the ring diameter was smaller than a certain value, no buckling occurred, similar to what had been stated by Evan-Iwanowski et al [29]; (ii) if the ring diameter was between two prescribed values, the buckling occurred as gradual formation of a dimple of reversed curvature; (iii) if the ring diameter was between a certain value and the diameter of the shell, then the buckling occurred with an abrupt change in shape. No comment was made about the probability of no-buckling via the appropriate choice of the thickness higher than a certain value depending on a set of values for the ring diameter and other dimensions of the shell.

The present study has been the third examination of this subject. The snap-through buckling behaviour of rigid vinyl polyethylene shallow simply-supported and clamped spherical shells subjected to the static ring loads is investigated numerically. The finite difference and Newton-Raphson Method are used. The load-deflection diagrams corresponding to various values of thickness, depth and ring diameter of the shell with simply supported and clamped edges are drawn and interpreted. The main contributions of this study to the relevant literature are: (i) extension of the range of the apical deflections whereby further characteristics of the load-deflection diagrams are revealed; (ii) determination of a certain value of thickness, depending on chosen ring diameter and depth of the shell, above which no snap-buckling occurs for a clamped spherical shell, the load-deflection diagram of which generally contains only one summit (upper-limit point), (iii) determination of a certain value of thickness of a simply-supported spherical shell, above which the first summit of the load-deflection diagram (which generally contains two summits) disappears, (accordingly; the appropriate choice of the thickness can change the snap-buckling loads and deflections considerably and, 
therefore, design criteria of such structures in a significant way.), (iv) the variations of the stress resultants, displacements along a meridian of the shell and deformed shapes of the shell corresponding to the various stages of the deformation (before, during and after the snapthrough buckling), which are essential for engineers dealing with the design of such structures, and which have not been encountered in the relevant literature.

In Section 2, the geometrical details, definitions of stress-resultants, equations of equilibrium, kinematic equations, constitutive relations and reduced form of the governing equations are given. The discretization of the governing equations by using the finite difference and the application of the Newton-Raphson Method are presented in Section 3. In Section 4, the effect of the variations of the ring diameter, thickness and depth of the shallow spherical shells, subjected to the ring loads, to the snap-through behaviour are investigated via the loaddeflection diagrams for both of the support conditions. The concluding remarks are presented in Section 5.

\section{GEOMETRY AND GOVERNING EQUATIONS OF SHELL OF REVOLUTION}

The geometry of a shell element of revolution before the deformation is represented in Figure $(1 \mathrm{a}, 1 \mathrm{~b})[31]$. In the figure, the two basic curvatures are specified $\left(1 / r_{1}\right.$ and $\left.1 / r_{2}\right)$ where $r_{1}$ is meridional radius of curvature and $r_{2}$ is circumferential radius of curvature, respectively. $r$ and $\mathrm{y}$ are the radial and vertical coordinates of a point on the surface; $s$ is the meridional arc length; $\phi$ and $\theta$ are the meridional and parallel central angles, respectively.

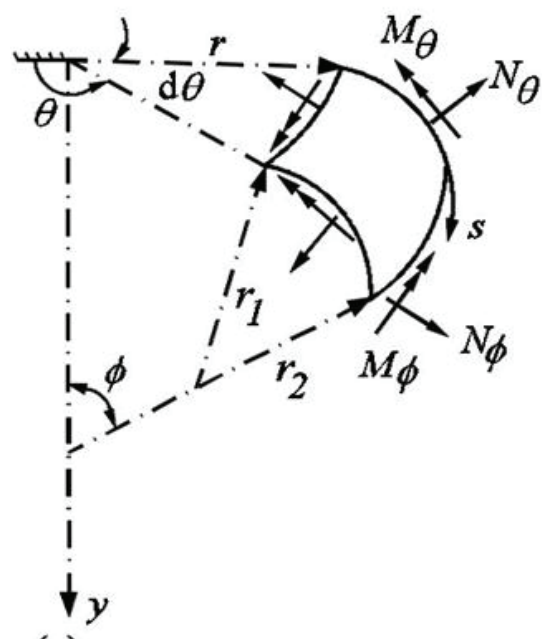

(a)

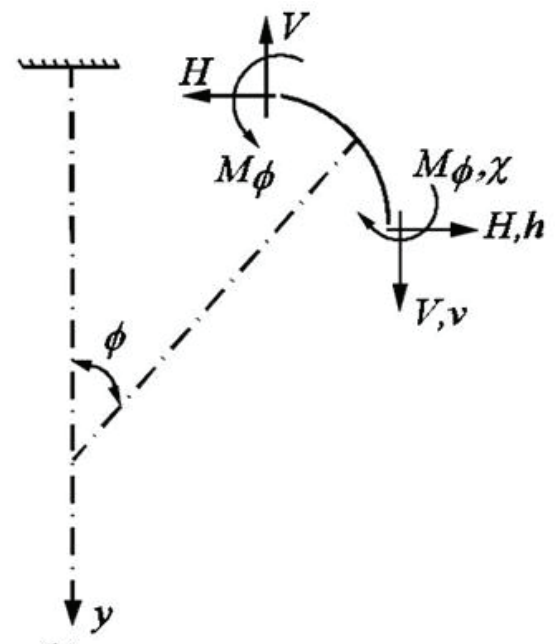

(b)

Figure 1 - The geometry of a shell element of revolution before the deformation.

The following basic relations are established [32]:

$d s=r_{1} d \phi, r_{2}=\frac{r}{\sin \phi}, d s^{2}=d r^{2}+d y^{2}, \frac{d y}{d r}=\tan \phi$ 


\subsection{Stress Resultants}

The stress resultants per unit length of the undeformed midsurface are defined by

$N_{\phi}=\int_{-t / 2}^{+t / 2} \sigma_{\phi \phi}\left(1+\frac{z}{r_{2}}\right) \mathrm{d} z, N_{\theta}=\int_{-t / 2}^{+t / 2} \sigma_{\theta \theta}\left(1+\frac{z}{r_{1}}\right) \mathrm{d} z, Q=\int_{-t / 2}^{+t / 2} \sigma_{\phi z}\left(1+\frac{z}{r_{2}}\right) \mathrm{d} z$,

$M_{\phi}=\int_{-t / 2}^{+t / 2} \sigma_{\phi \phi}\left(1+\frac{z}{r_{2}}\right) z \mathrm{~d} z, M_{\theta}=\int_{-t / 2}^{+t / 2} \sigma_{\theta \theta}\left(1+\frac{z}{r_{1}}\right) z \mathrm{~d} z$

where $\sigma_{\phi \phi}, \sigma_{\theta \theta}, \sigma_{z z}$ and $\sigma_{\phi z}$ are meridional stress, circumferential stress, transverse normal stress and transverse shear stress components, respectively; $N_{\phi}$ and $N_{\theta}$ are the meridional and circumferential forces, respectively; $Q$ is the transverse shear force; $M_{\phi}$ and $M_{\theta}$ are meridional and circumferential bending moments, respectively; $z$ is the normal distance from the midsurface and $t$ is the thickness of the undeformed shell. The positive signs of the stress resultants are shown in Figure (1b).

It is generally more convenient to study horizontal and vertical components; $H$ and $V$, respectively. The corresponding relations are as follows:

$V=N_{\phi} \sin \phi-Q \cos \phi, H=N_{\phi} \cos \phi+Q \sin \phi$.

where $V$ and $H$ are vertical and horizontal force resultants acting per unit length of the midsurface curve, respectively [32].

\subsection{Equilibrium Equations}

The equilibrium equations for axisymmetric deformation of a shell of revolution are as follows [30, 32]:

$\frac{\mathrm{d}}{\mathrm{d} s}(r V)=-r P_{V}, \quad \frac{\mathrm{d}}{\mathrm{d} s}(r H)=N_{\theta}-r P_{H}$,

$\frac{d}{\mathrm{~d} s}\left(r M_{\phi}\right)=r M_{\theta} \cos \phi+r H \sin \phi-r V \cos \phi$.

where $P_{V}$ and $P_{H}$ are vertical and horizontal surface loads acting per unit midsurface area of the undeformed shell, respectively [32].

\subsection{Kinematic Equations}

Displacements occurring at a point on the midsurface of the shell can be defined with the radial and vertical displacement components, $h$ and $v$ respectively, shown in Figure (1b). Meridional and circumferential strains $\left(e_{\phi}, e_{\theta}\right)$ in distance $z$ from the midsurface, respectively, are 
$e_{\phi}=\frac{\varepsilon_{\phi}+z \kappa_{\phi}}{1+\frac{z}{r_{1}}}, e_{\theta}=\frac{\varepsilon_{\theta}+z \kappa_{\theta}}{1+\frac{z}{r_{2}}}$

where $\varepsilon_{\phi}$ and $\varepsilon_{\theta}$ are meridional and circumferential midsurface strain measures, respectively; $\kappa_{\phi}$ and $\kappa_{\theta}$ meridional and circumferential curvature change measures, respectively. Equations (5) are valid for small strains. The linear measures used in equations (5) are:

$\varepsilon_{\phi}=\frac{\mathrm{d} h}{\mathrm{~d} s} \cos \phi+\frac{\mathrm{d} v}{\mathrm{~d} s} \sin \phi, \varepsilon_{\theta}=\frac{h}{r}, \kappa_{\phi}=\frac{\mathrm{d} \chi}{\mathrm{d} s}, \kappa_{\theta}=\frac{\chi \cos \phi}{r}$.

On the assumption that transverse shear deformation can be neglected, the rotation of the normal to the midsurface is equal to $\chi$.

\subsection{Constitutive Equations}

The stress component normal to the shell surface $\sigma_{z z}$ is assumed to be small compared to the stresses tangent to the surface $\left(\sigma_{z z} \ll \sigma_{\phi \phi}, \sigma_{\theta \theta}\right)$. Three dimensional constitutive equations for an isotropic, elastic shell after neglection of the transverse shear stress are written as follows:

$E e_{z}=-\vartheta\left(\sigma_{\phi \phi}+\sigma_{\theta \theta}\right), \sigma_{\phi \phi}=\frac{E}{1-\vartheta^{2}}\left(e_{\phi}+\vartheta e_{\theta}\right), \sigma_{\theta \theta}=\frac{E}{1-\vartheta^{2}}\left(e_{\theta}+\vartheta e_{\phi}\right)$.

A thin shell assumption is used $\left(\frac{t}{r_{1}} \ll 1, \frac{t}{r_{2}} \ll 1\right)$, therefore the terms including $\frac{z}{r_{i}}$ are considered to be negligibly small in comparison with unity in Eqs. (2) [23]. The corresponding two dimensional constitutive equations are

$N_{\phi}=\frac{E t}{1-\vartheta^{2}}\left(\varepsilon_{\phi}+\vartheta \varepsilon_{\theta}\right), \quad N_{\theta}=\frac{E t}{1-\vartheta^{2}}\left(\varepsilon_{\theta}+\vartheta \varepsilon_{\phi}\right)$,

$M_{\phi}=E t c^{2}\left(\kappa_{\phi}+\vartheta \kappa_{\theta}\right), M_{\theta}=E t c^{2}\left(\kappa_{\theta}+\vartheta \kappa_{\phi}\right)$.

where $E$ and $v$ are the elastic modulus and Poisson's ratio, respectively, and

$c^{2}=\frac{t^{2}}{12\left(1-\vartheta^{2}\right)}$

\subsection{Fundamental Equations of a Shell of Revolution}

The fundamental differential equations for a shell of revolution are reduced to a first order matrix system [32]. The general form of these equations is

$\left\{\frac{\mathrm{df}}{\mathrm{d} s}\right\}=[\mathrm{A}]\{\mathrm{f}\}+\{N(\mathrm{f})\}+\{\mu\}$

where 
$\{\mathrm{f}\}$ : the vector that contains the stress-resultants and displacements which is described as

$\{\mathrm{f}\}^{T}=\left[\begin{array}{llllll}r M_{\phi} & r H & r V & \chi & h & v\end{array}\right]$,

$[A]:$ the coefficient matrix which is described as

$$
[A]=\left[\begin{array}{cccccc}
\frac{\vartheta \cos \phi}{r} & \sin \phi & -\cos \phi & \frac{E t^{3} \cos ^{2} \phi}{12 r} & 0 & 0 \\
0 & \frac{\vartheta \cos \phi}{r} & \frac{\vartheta \sin \phi}{r} & 0 & \frac{E t}{r} & 0 \\
0 & 0 & 0 & 0 & 0 & 0 \\
\left(r E t c^{2}\right)^{-1} & 0 & 0 & \frac{-\vartheta \cos \phi}{r} & 0 & 0 \\
0 & \frac{1-\vartheta^{2}}{r E t} \cos ^{2} \phi & \frac{1-\vartheta^{2}}{r E t} \cos \phi \sin \phi & -\sin \phi & \frac{-\vartheta \cos \phi}{r} & 0 \\
0 & \frac{1-\vartheta^{2}}{r E t} \cos \phi \sin \phi & \frac{1-\vartheta^{2}}{r E t} \sin ^{2} \phi & -\cos \phi & \frac{-\vartheta \sin \phi}{r} & 0
\end{array}\right]
$$

$\{N(\mathrm{f})\}$ : the vector that contains non-linear terms which is described as

$$
\{N(\mathrm{f})\}=\left[\begin{array}{c}
\chi(r H \cos \phi+r V \sin \phi) \\
0 \\
0 \\
0 \\
-\chi^{2} \cos \phi / 2 \\
-\chi^{2} \sin \phi / 2
\end{array}\right]
$$

$\{\mu\}$ : the vector of loading terms which is described as

$$
\{\mu\}^{T}=\left[\begin{array}{llllll}
0 & -r P_{H} & -r P_{V} & 0 & 0 & 0
\end{array}\right] .
$$

The terms (11), (12), (13) and (14) are inserted into equation (10) and expressed explicitly as follows [30]:

$$
\begin{aligned}
&\left(r M_{\phi}\right)_{, s}=\left(\frac{\vartheta \cos \phi}{r}\right) r M_{\phi}+\sin \phi r H-\cos \phi r V+\frac{E t^{3} \cos ^{2} \phi}{12 r} \chi \\
&+\chi(r H \cos \phi+r V \sin \phi), \\
&(r H)_{, s}=\left(\frac{\vartheta \cos \phi}{r}\right) r H+\left(\frac{\vartheta \sin \phi}{r}\right) r V+\frac{E t}{r} h, \\
&(r V)_{, s}=0, \\
& \chi_{, s}=\frac{M_{\phi}}{E t c^{2}}-\frac{\vartheta \cos \phi}{r} \chi, \\
& h_{, s}=\frac{1-\vartheta^{2}}{r E t} \cos ^{2} \phi r H+\frac{1-\vartheta^{2}}{r E t} \sin \phi \cos \phi r V-\sin \phi \chi-\frac{\vartheta \cos \phi}{r} h-\frac{\chi^{2} \cos \phi}{2},
\end{aligned}
$$


$v_{, S}=\frac{1-\vartheta^{2}}{r E t} \sin \phi \cos \phi r H+\frac{1-\vartheta^{2}}{r E t} \sin ^{2} \phi r V+\cos \phi \chi-\frac{\vartheta \sin \phi}{r} h-\frac{\chi^{2} \sin \phi}{2}$.

In this study, for the mathematical structure of the manuscript, Reference [32] has been used which was one of the pioneering works on this field. There are, of course, new studies providing improvements on this subject by relaxing the restrictions on rotations and deformations (e.g. by taking into account the transverse normal and transverse shear strains) and by using higher order constitutive relations. If a rubber-like material, for example, which can undergo very large elastic strains, had been considered, then it would have been inevitable to deal with an advanced shell theory, developed recently.

\section{SOLUTION METHOD}

In this section, the fundamental differential equations for a shell of revolution, given in the previous section, are applied for the shallow spherical shell. If the ratio of the rise of the midsurface of the shell at the centre to the base diameter is smaller than $1 / 8$, the concerning spherical shell is called "shallow" [33]:

$\eta=\frac{B}{2 a} \leq \frac{1}{8}$

where $\eta, B$ and $a$ are the parameter of depth, the rise of the midsurface of the shell at the centre and the base radius, respectively, as shown in Figure 2. The spherical shell subjected to a static ring load, shown in Figure 2, is divided into $(n n-1)$ finite segments, corresponding to $n n$ nodes where the first node is located at a clamped support or a simple support while the final node is located at the apex, with the step length of $\Delta s$. There is a uniformly distributed ring load with the resultant $P$ at the point $\ell \ell$ of the shell where $r_{0}$ is the radius of the ring before the deformation. $R$ is the radius of the undeformed shell. $\phi_{0}$ and $\phi_{m}$ are the meridional angles at the application point of the ring load and at the support, respectively.

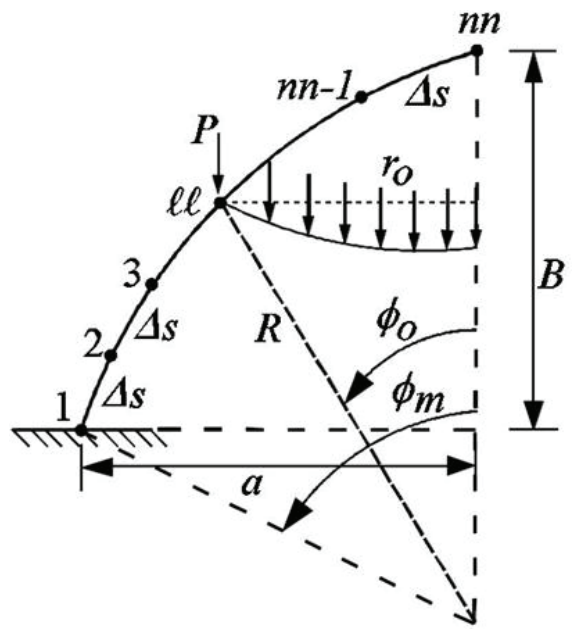

Figure 2 - Shell divided into finite segments. 
The derivatives seen in Eqs. (15)-(20) are expressed by using the forward difference formulas for any point $(i)$ and the following nonlinear algebraic equations are obtained:

$$
\begin{aligned}
& -M_{i+1}+M_{i}\left(1+\frac{\Delta \vartheta \cos \phi_{i}}{r_{i}}-\frac{\Delta \cos \phi_{i}}{r_{i}}\right)+\Delta \sin \phi_{i} H_{i}-\Delta V_{i} \cos \phi_{i}+\frac{\Delta E t^{3} \cos ^{2} \phi_{i}}{12 r_{i}^{2}} \chi_{i}+ \\
& \Delta \chi_{i}\left(H_{i} \cos \phi_{i}+V_{i} \sin \phi_{i}\right)=0, \\
& -H_{i+1}+H_{i}\left(1+\frac{\Delta \vartheta \cos \phi_{i}}{r_{i}}-\frac{\Delta \cos \phi_{i}}{r_{i}}\right)+\frac{\Delta \vartheta \sin \phi_{i}}{r_{i}} V_{i}+\frac{\Delta E t}{r_{i}^{2}} h_{i}=0, \\
& -V_{i+1}+V_{i}\left(1-\frac{\Delta \cos \phi_{i}}{r_{i}}\right)=0, \\
& -\chi_{i+1}+\chi_{i}\left(1-\frac{\Delta \vartheta \cos \phi_{i}}{r_{i}}\right)+\frac{\Delta M_{i}}{E t c^{2}}=0, \\
& -h_{i+1}+h_{i}\left(1-\frac{\Delta \vartheta \cos \phi_{i}}{r_{i}}\right)+\frac{\Delta\left(1-\vartheta^{2}\right) \cos ^{2} \phi_{i}}{E t} H_{i}+\frac{\Delta\left(1-\vartheta^{2}\right) \cos \phi_{i} \sin \phi_{i}}{E t} V_{i}-\Delta \sin \phi_{i} \chi_{i}- \\
& \Delta \chi_{i}^{2} \frac{\cos \phi_{i}}{2}=0, \\
& -v_{i+1}+v_{i}+\frac{\Delta\left(1-\vartheta^{2}\right) \sin \phi_{i} \cos \phi_{i}}{E t} H_{i}+\frac{\Delta\left(1-\vartheta^{2}\right) \sin ^{2} \phi_{i}}{E t} V_{i}-\Delta \cos \phi_{i} \chi_{i} \frac{\Delta \vartheta \sin \phi_{i}}{r_{i}} h_{i}- \\
& \quad \Delta \chi_{i}^{2} \frac{\cos \phi_{i}}{2}=0 .
\end{aligned}
$$

Equations (22)-(27) are employed between the points $(1, \ell \ell-2)$ of the shell. At the point $(\ell \ell-1)$, on the other hand, the equation related to the vertical force resultant, Eq.(24), is omitted in order to exclude the external force $P$ from the list of the unknows. Therefore, 5 equations are written for this point. Moreover, 5 equations are written at each node between the points $(\ell \ell, n n-1)$ including the point $(n n-1)$ due to the fact that the vertical force resultant is equal zero for these points which may easily be obtained from the equilibrium of the concerning segment. The numerical solution is derived by the Newton-Raphson method. In order not to encounter a problem of convergence in the vicinity of the region where the slope of the force-deflection curve is zero, the apical vertical displacement $v_{n n}$ is used in the input data instead of the ring load $P{ }^{\dagger}$ The initial guess of the state vector is taken to be zero vector $(\{f\}=\{0\})$, corresponding to the zero value of $v_{n n}$. As a criteria for the convergence of the successive approximations of the Newton-Raphson method, a vector corresponding to the energy level at the $(k)$ th iteration is defined as follows [30]:

$$
\left\|f^{(k)}(s)\right\|_{E}=\left(\left|r M_{\phi} \cdot \chi\right|+|r H . h|+|r V \cdot v|\right)
$$

\footnotetext{
$\dagger$ There have been advanced techniques, proposed by Memon and Su [34], Riks [35,36], Wempner [37], Polat and Calayır [38], Polat [39] to treat the convergence problem in the vicinity of the limit points of the force-deflection curves, which may unavoidably be used for relatively more complex problems than the one considered in the current study.
} 
The convergence at any iteration is considered to be achieved, if the relative difference $\Omega$, defined as

$\Omega=\frac{\left\|f^{(k+1)}(s)\right\|_{E}-\left\|f^{(k)}(s)\right\|_{E}}{\left\|f^{(k+1)}(s)\right\|_{E}}$

is smaller than a prescribed value. The boundary conditions at the apex $(n n)$ are

$\chi_{n n}=h_{n n}=0$.

The boundary conditions for a clamped edge are

$\chi_{1}=h_{1}=v_{1}=0$,

while the boundary conditions for a simply supported edge are

$M_{1}=h_{1}=v_{1}=0$.

$(5 n n+\ell \ell-7)$ equations written between the points $(1, n n-1)$ and 3 boundary conditions at the support represent a total equal to $(5 n n+\ell \ell-4)$ equations which is equal to the number of unknowns. The external force $P$ which is absent in the state vector can be computed by the help of the vertical equilibrium equation of the whole of the shell after the numerical solution of the unknowns at each level of the loading is obtained:

$P 2 \pi r_{0}+V_{1} 2 \pi a=0$.

where

$r_{0}=R \sin \phi_{0}, \quad a=R \sin \phi_{m}$.

where $R, r_{0}, \phi_{0}$ and $\phi_{m}$ denote the radius of the spherical shell, the radius of the ring load, the meridional angle at the application point of the ring load and the meridional angle at the support, respectively.

\section{NUMERICAL EXPERIMENTS}

The effect of the variation of the diameter of the ring load, thickness and depth of the shell on the axially symmetrical ring load versus the deflection curves at the point of application of the load for two types of supports (clamped edge and simply supported edge) are investigated by several numerical experiments. Additionally, the variations of the stressresultants and deformed shapes are investigated corresponding to various stages of the loading; before, during and after the snap-through buckling (where the slope of the loaddeflection curve is zero).

Computer program used in the numerical study has been coded by the authors in the package FTN90. 
In this study, a non-dimensional load $P^{*}$, the resultant of the distributed ring load, acting at any height of the shell, and a nondimensional deflection $v^{*}$ at the application point of the ring load are defined as follows:

$P^{*}=\frac{P R}{E t^{3}}, \quad v^{*}=\frac{v_{i}}{t}$.

where $v_{i}$ is the deflection at the application point of the ring load.

In order to demonstrate the accuracy of the prepared computer program, the numerical results obtained by using the computer program prepared in the present study are compared with those obtained by Evan-Iwanowski et al. [29] via an experimental study on die-pressed plastic shallow shells made of rigid vinyl polyethylene (rigid PVC) with radii of $254 \mathrm{~mm}$. The results of the comparison are shown in Figure 3. A sufficient agreement is observed.

The curves of the non-dimensional load $P^{*}$ versus the non-dimensional deflection $v^{*}$ obtained in the numerical experiments for various values of the diameter of the ring load, the shell thickness and the parameter of the depth for clamped edge and simply supported edge are shown in Figures (3-8).

It can be seen from Figure 3 that there is no snap-buckling for $d \leq 15.0492 \mathrm{~mm}$. The curve corresponding to $d=15.0492$ is indicated by a solid red line. The curves indicated by blue lines are for $d=12.7 \mathrm{~mm}$ and those indicated by black lines are for $d=25.4 \mathrm{~mm}$. As the ring diameter $d$ is increased, both of the buckling load and buckling deflection increase, as well

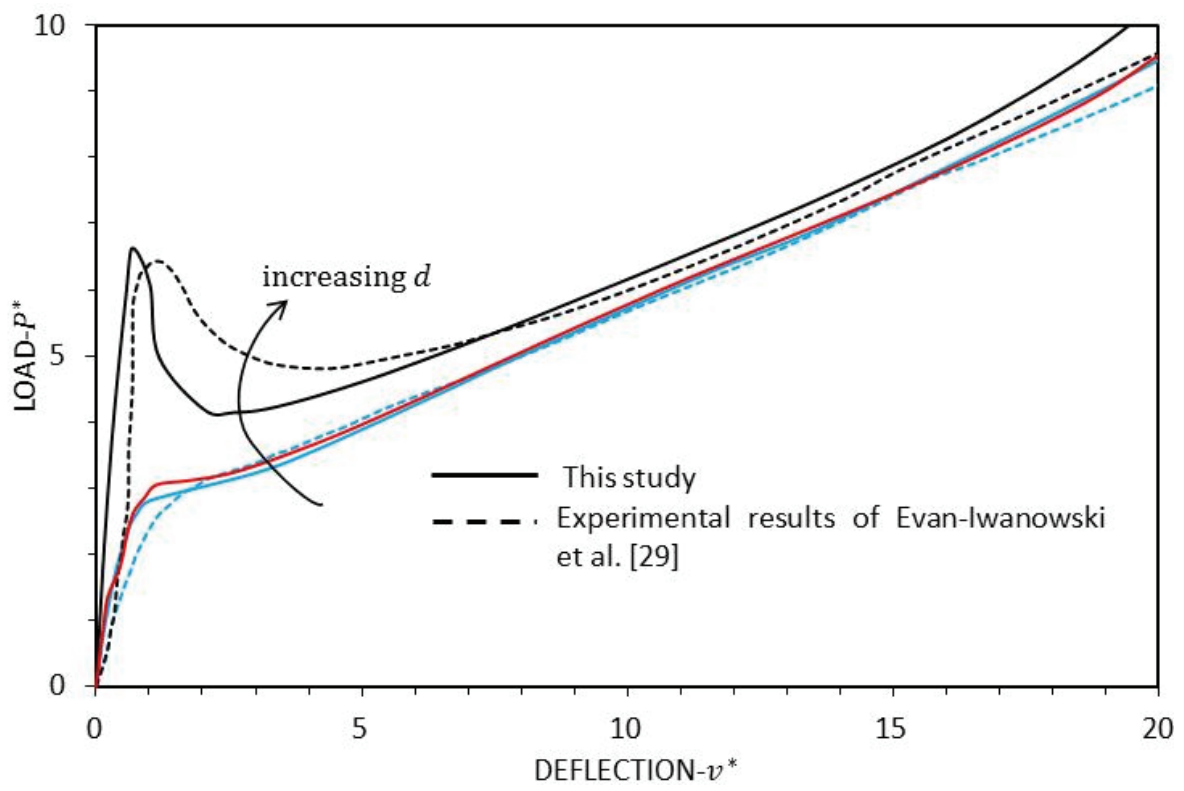

Figure 3 - Comparison of the load-deflection curves obtained in this study with those corresponding to the experiments performed by Evan-Iwanowski et al. [29] for clamped edge shallow shell for $\eta=0.0618, t=0.3810 \mathrm{~mm}, d=12.7 ; 15.0492 ; 25.4 \mathrm{~mm}$. 
Figure 4 shows the load-deflection curves of the clamped shallow spherical shells for various values of $t$, corresponding to the given values of $d$ and $\eta$. Accordingly; for (i) $t=$ $0.3810 \mathrm{~mm}$, the load-deflection curve has two peaks (upper limit points) the first of which controls the buckling; (ii) $t=0.7620 \mathrm{~mm}$, the second summit is seen to disappear, leaving a unique summit; (iii) $t=1.0660 \mathrm{~mm}$, there is no summit, meaning that no snap-buckling occurs for this value of the thickness and those greater than this value. As the thickness $t$ is decreased, both of the buckling load and buckling deflection are increased, as well. Therefore; an appropriate choice of the thickness, depending on the values $d$ and $\eta$, can prevent the likelihood of snap-buckling completely.

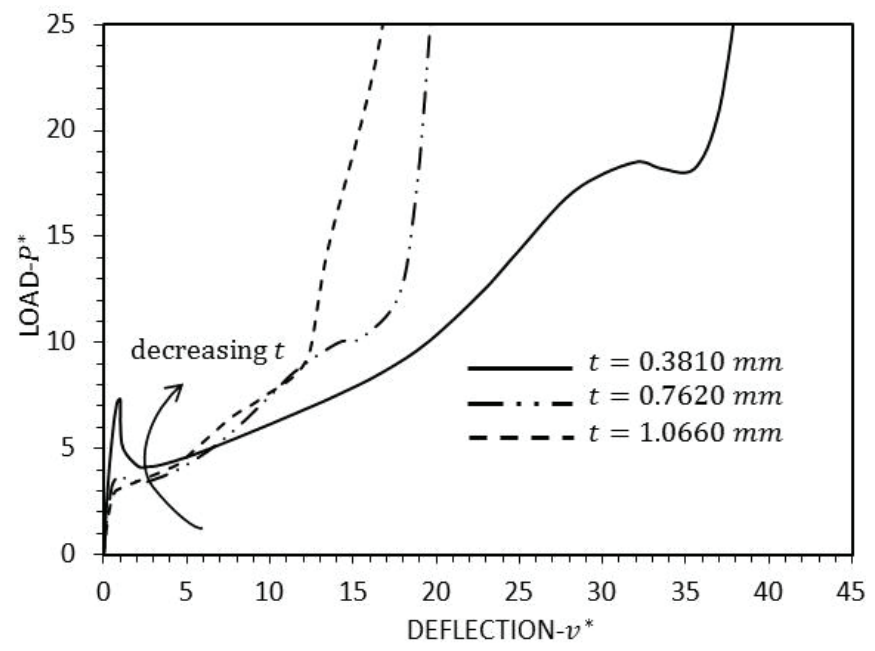

Figure 4 - Load-deflection curves for different values of t for clamped edge shallow shell for $\eta=0.0618, d=25.4 \mathrm{~mm}$.

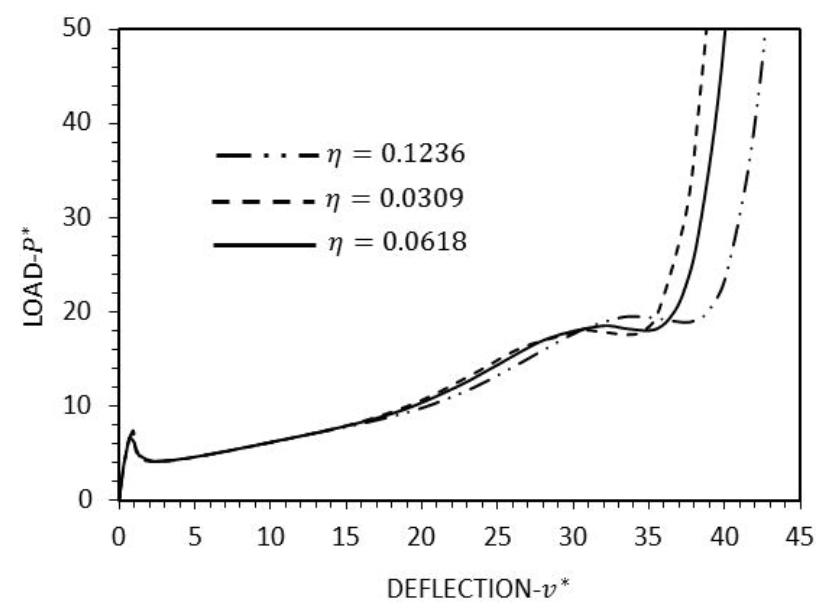

Figure 5 - Load-deflection curves for various values of $\eta$ for clamped edge shallow shell for $d=25.4 \mathrm{~mm}, t=0.3810 \mathrm{~mm}$. 
Figure 5 illustrates the effect of $\eta$ on the concerning behaviour of the clamped shallow spherical shells for the given values of $t$ and $d$. Extending the range of the values of $v^{*}$, second summits in the load-deflection diagrams are observed at about $30<v^{*}<35$, which were not seen in the previous studies. The first summits, therefore the buckling loads and buckling deflections, are observed to be unaffected by the variation of $\eta$.

The load-deflection curves, shown in Figure 6, of the simply supported shallow spherical shells for various values of $d$, corresponding to the given values of $t$ and $\eta$, have two summits for $d>14.9314 \mathrm{~mm}$, in which cases the first summit dominate the buckling. For any curve corresponding to $d \leq 14.9314 \mathrm{~mm}$, there is only one summit. This phenomenon yields that a choice of the ring diameter $d$ smaller than or equal to $14.9314 \mathrm{~mm}$, corresponding to the given values of $t$ and $\eta$, increases the buckling loads and buckling deflections remarkably.

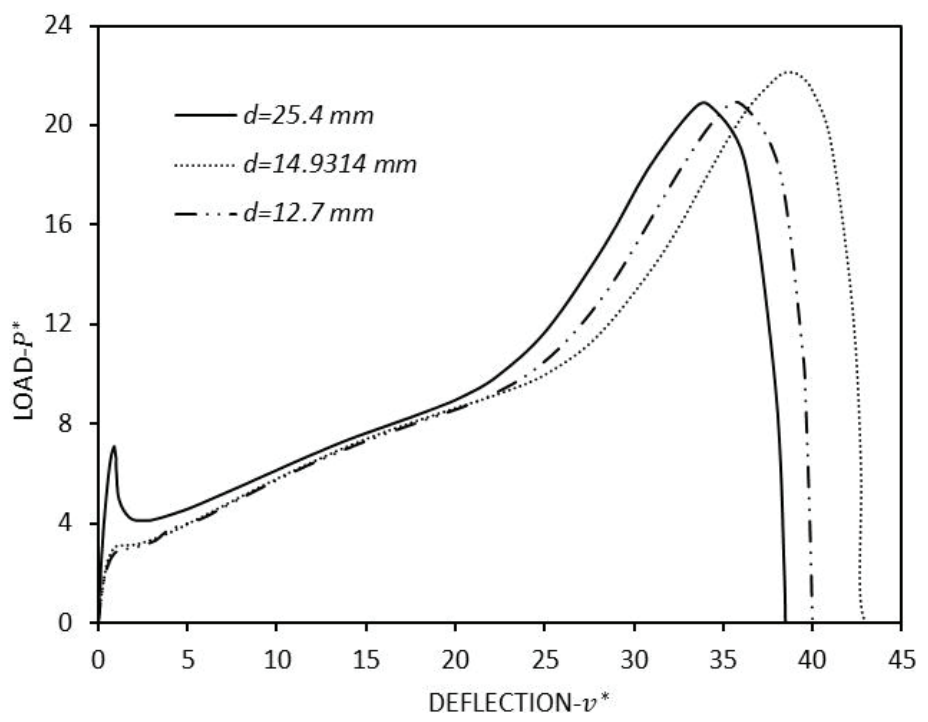

Figure 6 - Load-deflection curves for various values of d for simply supported shallow shell for $t=0.3810 \mathrm{~mm}, \eta=0.0668$.

The load-deflection curves, shown in Figure 7, of the simply supported shallow spherical shells for various values of $t$, corresponding to the given values of $d$ and $\eta$, have two peaks, except the one belonging to $t=1.1307 \mathrm{~mm}$ (in which case, there is only one summit), similar to what was observed in Evan-Iwanowski et al. [29]. This phenomenon suggests that a choice of the thickness $t$ greater than or equal to $1.1307 \mathrm{~mm}$, corresponding to the given values of $d$ and $\eta$, increases the buckling loads and buckling deflections remarkably.

The load-deflection curves, shown in Figure 8, of the simply supported shallow spherical shells for various values of $\eta$, corresponding to the given values of $t$ and $d$, have two summits. Increasing the values of $\eta$ is seen to cause increase in the coordinates of the second summits considerably, but this has almost no effect on the first summit, which controls the buckling load and buckling deflection. 


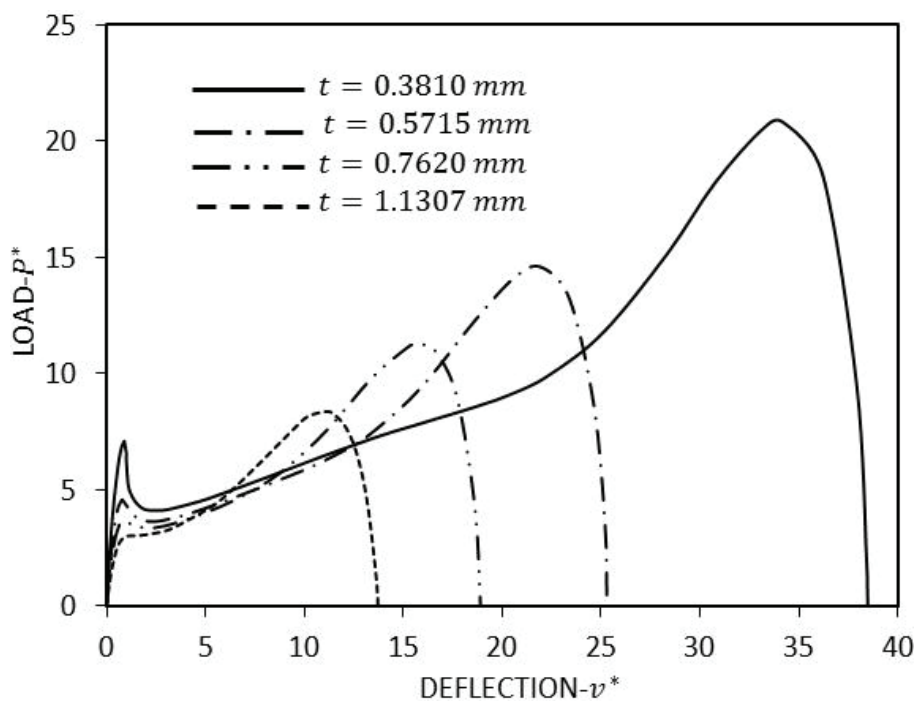

Figure 7 - Load-deflection curves for various values of t for simply supported shallow shell for $\eta=0.0668, d=25.4 \mathrm{~mm}$.

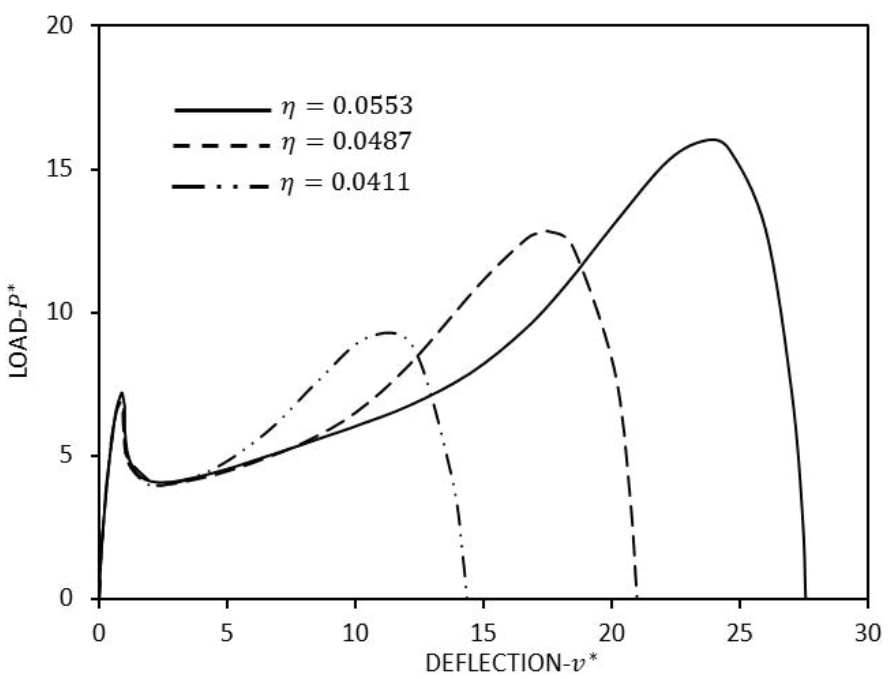

Figure 8 - Load-deflection curves for various values of $\eta$ for simply supported shallow shell for $t=0.6350 \mathrm{~mm}, d=25.4 \mathrm{~mm}$.

Comparison of some of the load-deflection curves corresponding to the simply supported and clamped shallow shells are shown in Figure 9 and Figure 10, by varying $d$ and $t$, respectively. Interestingly, the load-deflection curves corresponding to the concerning supports almost coincide in the neighbourhood of the first summits. The load-deflection diagrams corresponding to the simply supported shallow spherical shells are seen to, generally, contain 
two summits, the first of which can disappear via the appropriate choices of $t, d$ and $\eta$, as mentioned. The second summits in the load-deflection diagrams corresponding to the clamped shallow spherical shells are observed to be realized only at relatively high values of the deflection. For the values of $d=12.7 \mathrm{~mm}, t=0.3810 \mathrm{~mm}$ and $\eta=0.0668$, the first summit disappears, seen in Figure 9.

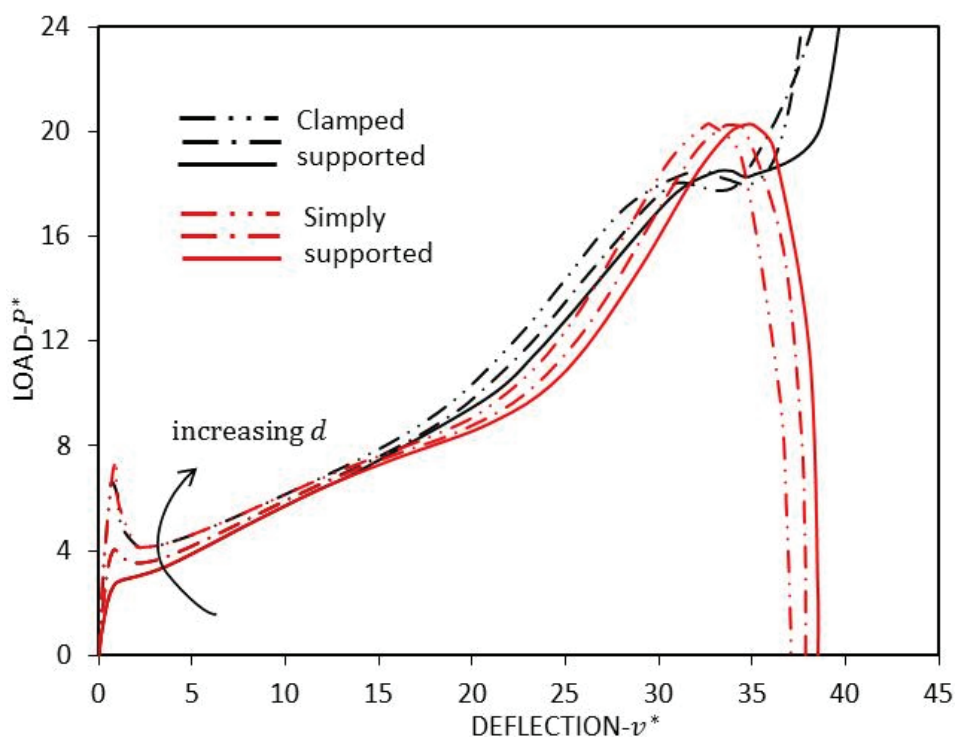

Figure 9 - Comparison of the results corresponding to clamped and simply supported for different values of $d$ for $\eta=0.0618, t=0.3810 \mathrm{~mm}, d=12.7 ; 19.1 ; 25.4 \mathrm{~mm}$.

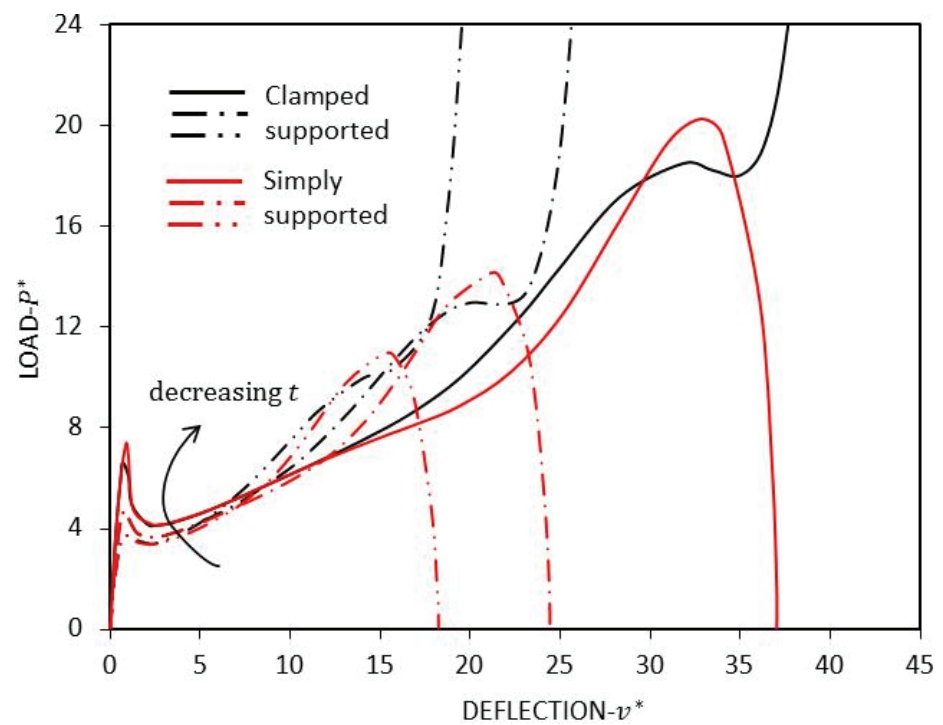

Figure 10 - Comparison of the results corresponding to clamped and simply supported for different values of $t$ for $\eta=0.0618, d=25.4, t=0.3810 ; 0.5715 ; 0.7620 \mathrm{~mm}$. 
In order to see the variations of the stress-resultants, displacements and the deformed shapes of a clamped shallow spherical shell along the arc length $s$ during the various stages of the loading, the graphs of the stress-resultants and the deformed shapes of the shell corresponding to various values of $v^{*}$ (before the buckling, during the buckling and after the buckling corresponding to the load-deflection diagram seen in Figure 3 for $d=25.4 \mathrm{~mm}, t=$ $0.3810 \mathrm{~mm}, \eta=0.0618$ ) are shown in Figures 11-16. $v_{c r}^{*}$ is the deflection of the shell (at the level of the ring) when the buckling occurs ${ }^{*} s^{*}$, seen in Figures 11-16, is the nondimensional meridian length such that $s^{*}$ is taken to be zero at the apex and equal to 1 at the support. The value of $s^{*}$ at any point $i$ of the meridian can be checked to be determined as

$s^{*}=\frac{1}{(n n-1)}[(n n-1)-(i-1)]$

In Figure 11; the diagrams of the meridional bending moment $M$ along a meridian corresponding to the various stages of the deformation (namely; before the buckling, during the buckling and after the buckling) are shown for a given set of values of $d, t$ and $\eta$. An abrubt change in the magnitude and sign of $M$ due to the buckling is observed clearly. The apical moment (at $s^{*}=0$ ) should be considered primarily for the design of such a structure.

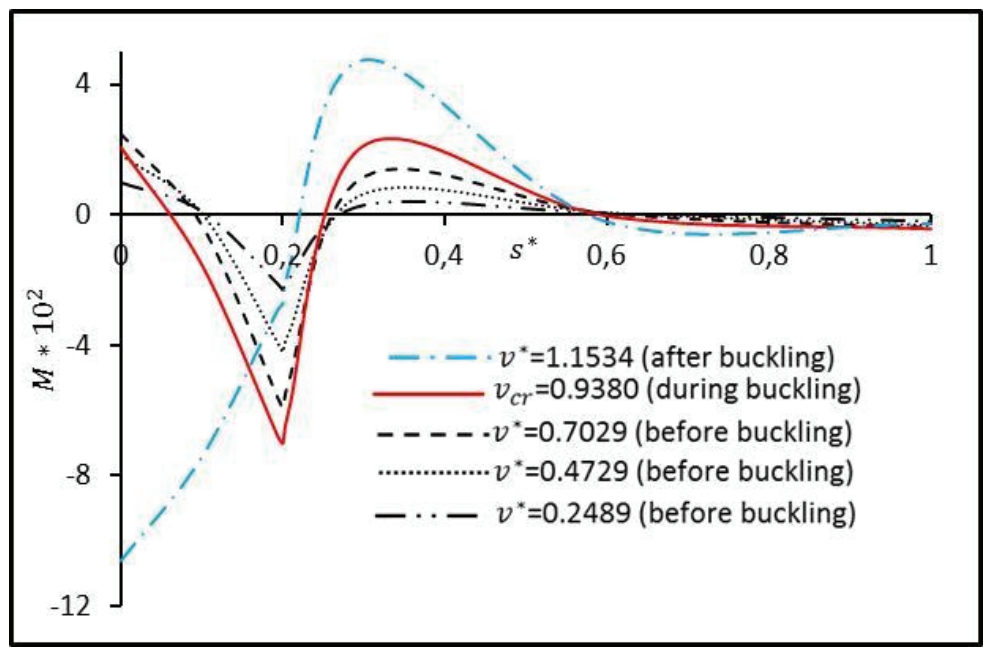

Figure 11 - The variation of the meridional moment $M$ along the meridian for various values of $v^{*}$ for $\eta=0.0618, d=25.4 \mathrm{~mm}, t=0.3810 \mathrm{~mm}$.

The diagrams of the horizontal force resultant $H$ along a meridian corresponding to the various stages of the deformation are shown for a given set of values of $d, t$ and $\eta$ in Figure 12. The values of $H$ are seen to be increasing drastically during and after the buckling. The apical $H$ value should be considered to be most essential value.

\footnotetext{
In other words, $v_{c r}^{*}$ is the deflection where the slope of the load-deflection diagram vanishes.
} 
The diagrams of the vertical force resultant $V$ along a meridian corresponding to the various stages of the deformation are shown for a given set of values of $d, t$ and $\eta$ in Figure 13. $V$ is seen to vanish between the apex and the ring, as expected. It attains its highest values in the neighbourhood of the ring.

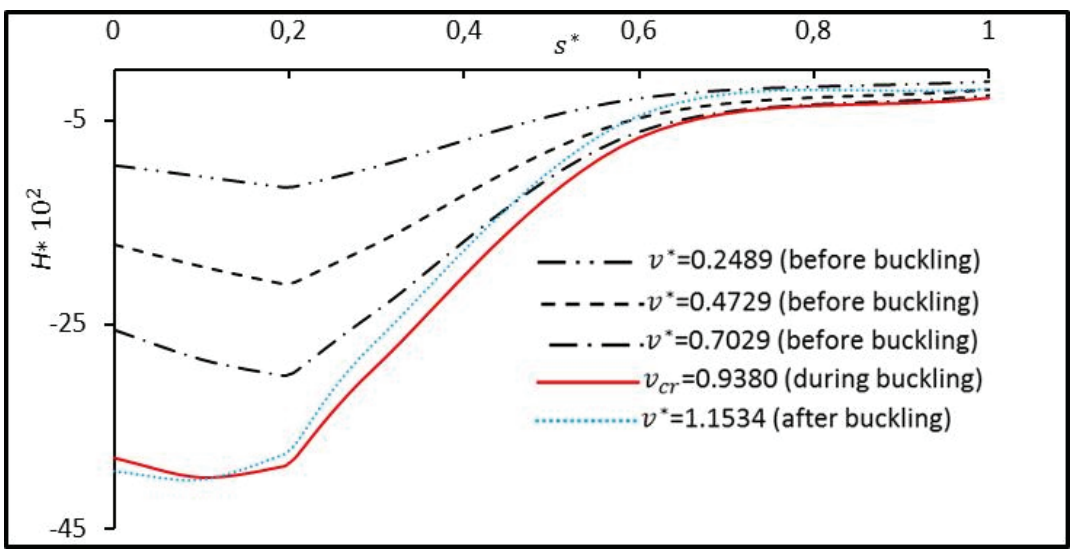

Figure 12 - The variation of the horizontal force resultant $H$ along the meridian for various values of $v^{*}$ for $\eta=0.0618, d=25.4 \mathrm{~mm}, t=0.3810 \mathrm{~mm}$.

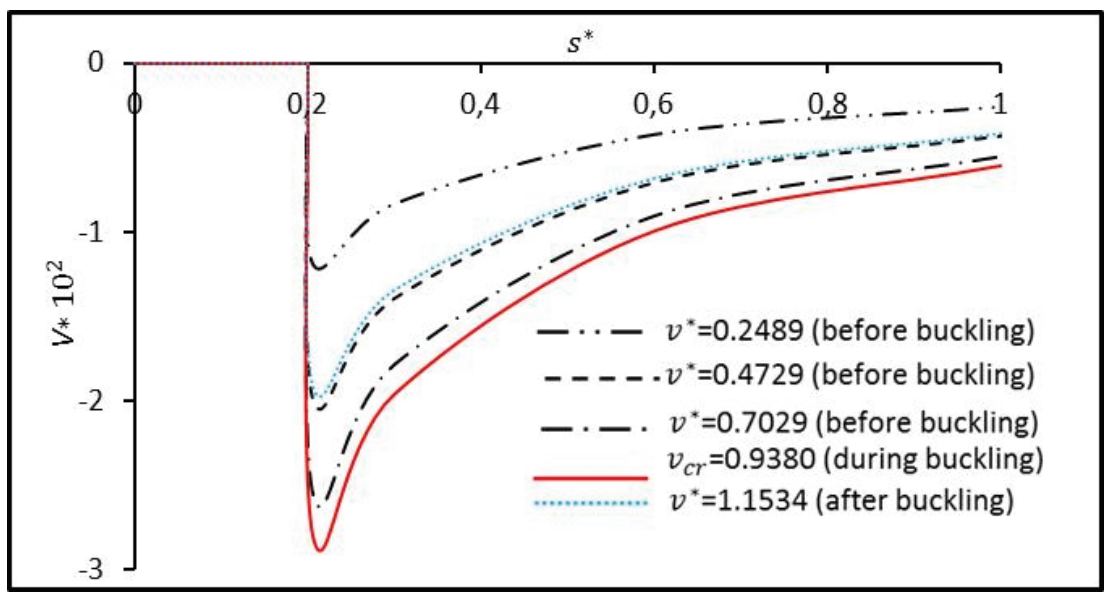

Figure 13 - The variation of the vertical force resultant $V$ along the meridian for various values of $v^{*}$ for $\eta=0.0618, d=25.4 \mathrm{~mm}, t=0.3810 \mathrm{~mm}$.

The diagrams of the rotation angle $\chi$ corresponding to the various stages of the deformation are shown in Figure 14 for a given set of values of $d, t$ and $\eta$. The rotation angle is zero at the apex, as a boundary condition. It reaches its maximum value in the neighbourhood of the ring, especially after the buckling. 
The diagrams of the horizontal displacement $h$ corresponding to the various stages of the deformation are shown in Figure 15 for a given set of values of $d, t$ and $\eta$. $h$ is zero at the apex, as expected. It reaches its highest values in the region of $0.5 \leq s^{*} \geq 0.6$.

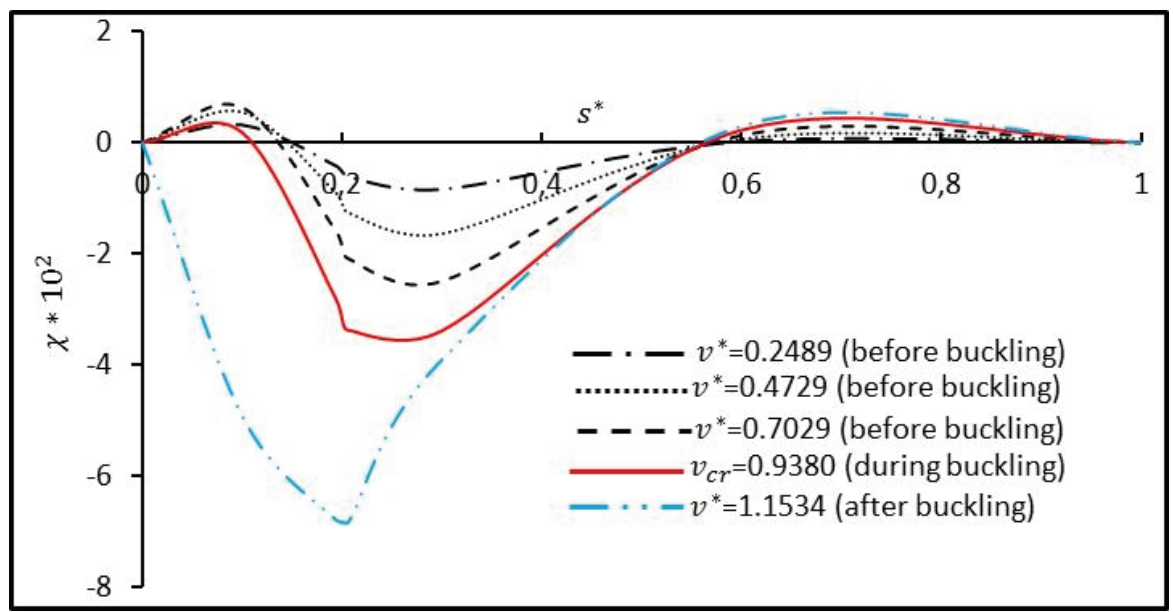

Figure 14 - The variation of the rotation $\chi$ of the normal to the midsurface for various values of $v^{*}$ for $\eta=0.0618, d=25.4 \mathrm{~mm}, t=0.3810 \mathrm{~mm}$.

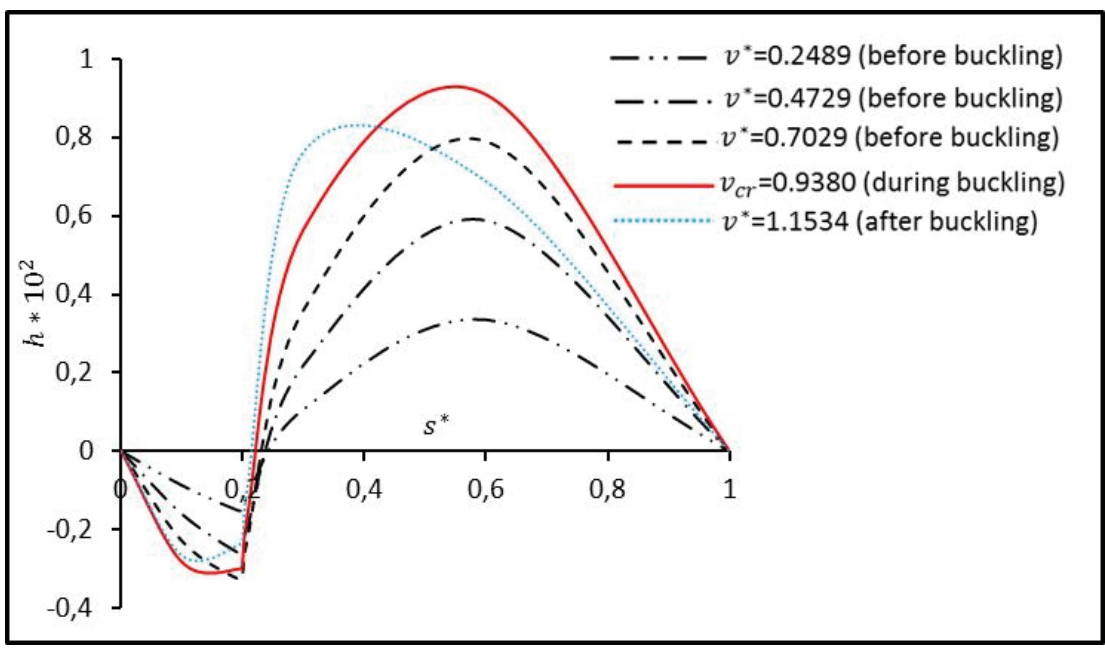

Figure 15 - The variation of the horizontal displacement $h$ for various values of $v^{*}$ for $\eta=$ $0.0618, d=25.4 \mathrm{~mm}, t=0.3810 \mathrm{~mm}$.

The diagrams of the vertical displacement $v$ corresponding to the various stages of the deformation are shown in Figure 16 for a given set of values of $d, t$ and $\eta$. The vertical 
displacement is seen to obtain its highest values at the apex. An abrupt increase in the values of $v$ is observed in the figure due to the buckling.

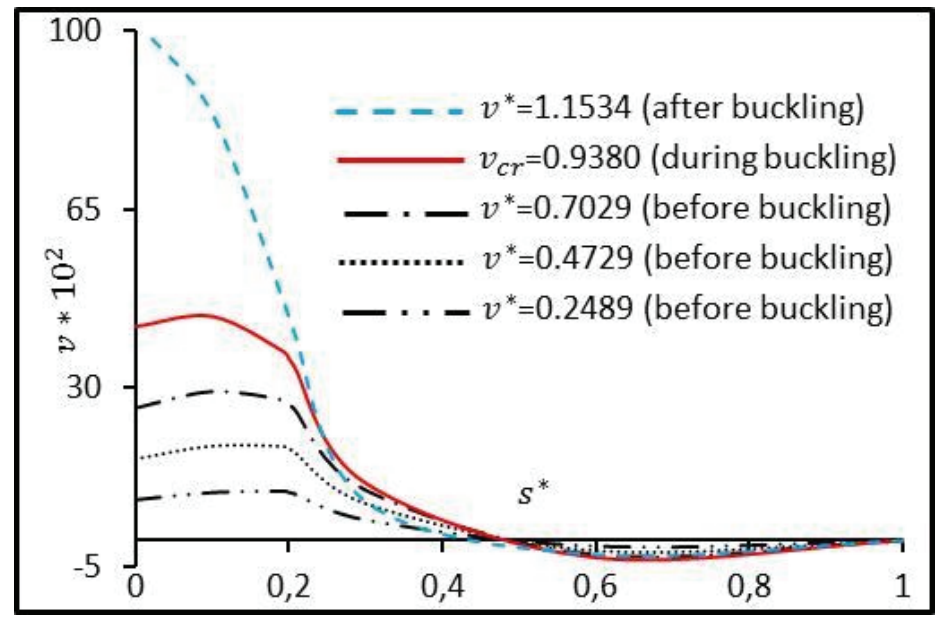

Figure 16 - The variation of the vertical displacement $v$ for various values of $v^{*}$ for $\eta=$ $0.0618, d=25.4 \mathrm{~mm}, t=0.3810 \mathrm{~mm}$.

Deformed shapes of a clamped shallow shell corresponding to various stages of load, namely those corresponding to before the buckling $\left(v^{*}<v_{c r}\right)$, during the buckling $\left(v^{*}=v_{c r}\right)$ and after the buckling $\left(v^{*}>v_{c r}\right)$ are shown in Figure 17. The auxiliary expressions are presented in Appendix A and Appendix B. A very drastic deformation can be observed after buckling.

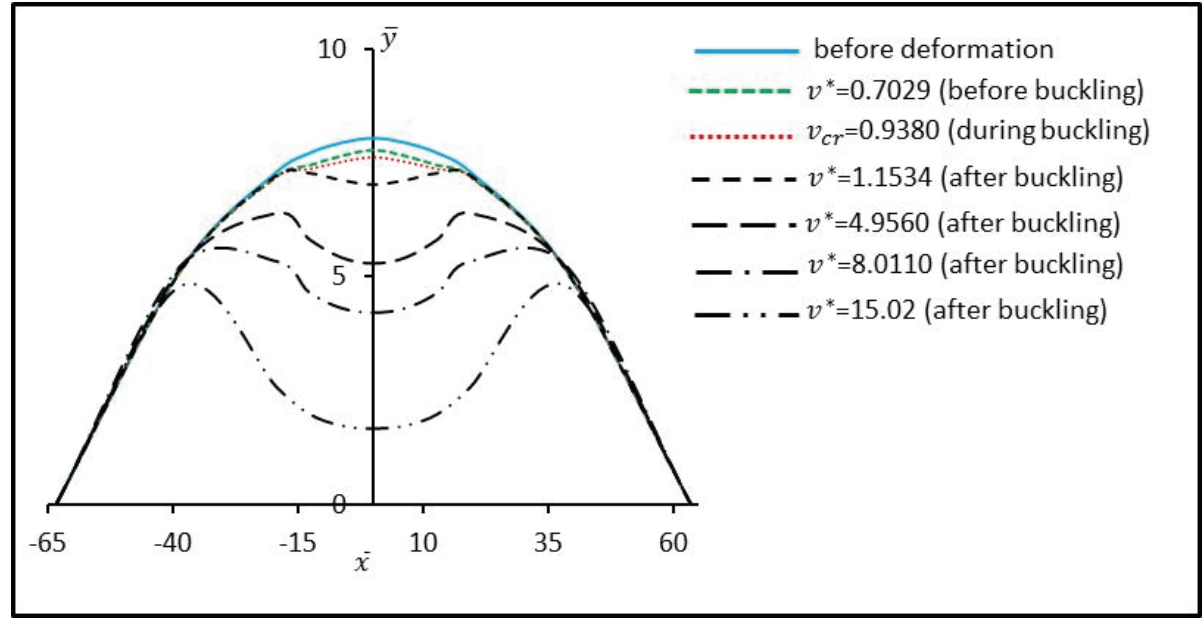

Figure 17 - Geometries of shallow spherical shell before, during and after deformation for clamped shell for $\eta=0.0618, d=25.4 \mathrm{~mm}, t=0.3810 \mathrm{~mm}$. 


\section{CONCLUSIONS}

According to the numerical results obtained for the rigid vinyl polyethylene shallow spherical shells subjected to the static ring loads, the following main conclusions can be drawn:

- The numerical results obtained in this study are sufficiently compatible with the results of the experimental study conducted by Evan-Iwanowski et al. [29].

- It is possible to prevent the probability of the snap-buckling of a clamped shallow spherical shell through the appropriate choices of the ring diameter, thickness and depth of the shell.

- It is possible to increase the snap-buckling loads and deflections considerably for a simply supported shallow spherical shell through the appropriate choices of the ring diameter, thickness and depth of the shell.

- As the thickness of a shell is decreased and/or the ring diameter is increased, both of the snap-buckling load and the snap-buckling deflection are increased.

- The snap-buckling loads and the snap-buckling deflections of the shallow spherical shells are not significantly affected by the variation of the depth of the shell.

- The second summits of the load-deflection diagrams of the clamped shallow spherical shells are revealed by extending the range of the apical deflections.

- For the design and/or analysis of such a structure; (i) the apical values of the deflection (vertical displacement), bending moment and the horizontal force, (ii) the values of the vertical force and the rotation angle in the neighbourhood of the ring should be considered primarily.

\section{Appendix A}

The coordinates $x$ and $y$ of a point of the midsurface of the shell before the deformation can be checked to be expressed as in terms of $R, \phi$ and $\phi_{m}$ as

$$
\begin{aligned}
x & =R \sin \phi, \\
y & =R\left(\cos \phi-\cos \phi_{m}\right),
\end{aligned}
$$

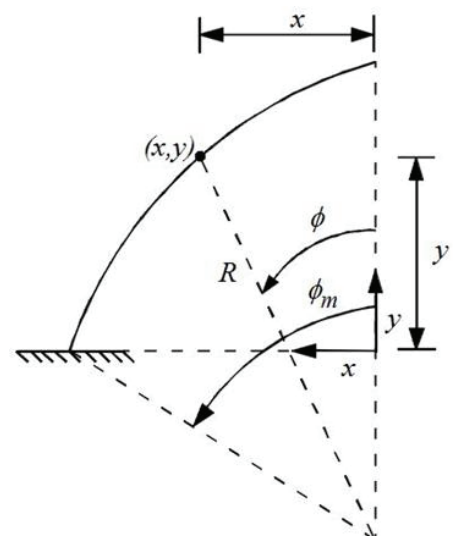

Figure 18 - Geometry of shell before deformation. 


\section{Appendix B}

Using the displacement components $h$ and $v$, the coordinates $\bar{x}$ and $\bar{y}$ of a point of the midsurface of the shell after the deformation can be checked to be expressed as

$$
\begin{aligned}
& \bar{x}=R \sin \phi+h, \\
& \bar{y}=R\left(\cos \phi-\cos \phi_{m}\right)-v .
\end{aligned}
$$

\section{References}

[1] Labisch, F. K., On the Axisymmetric Buckling Behavior of Incompressible Nonlinearly Elastic Spherical Shells. Z. Angew. Math. Mech., 86(7), 572-583, 2006.

[2] Pi, Y. L., Bradford, M. A. and Tin-Loi, F., Nonlinear Analysis and Buckling of Elastically Supported Circular Shallow Arches. International Journal of Solids and Structures, 44, 2401-2425, 2007.

[3] Guana, F. and Pastrone, F., Axisymmetric Instabilities for Elastic Conical Shells under Compressive End Loadings. Mathematics and Mechanics of Solids, 12, 164-182, 2007.

[4] Pi, Y. L., Bradford, M. A. and Tin-Loi, F., Non-linear In-plane Buckling of Rotationally Restrained Shallow Arches under a Central Concentrated Load. International Journal of Non-Linear Mechanics, 43, 1-17, 2008.

[5] Cai, J. and Feng, J., Buckling of Parabolic Shallow Arches When Support Stiffens under Compression. Mechanics Research Communications, 37, 767-471, 2010.

[6] Coman, C. D., Bifurcation Instabilities in Finite Bending of Circular Cylindrical Shells. International Journal of Engineering Science, 119, 249-264, 2017.

[7] Hutchinson, J. W., Buckling of Spherical Shells Revisited, Proceedings of the Royal Society A: Mathematical, Physical and Engineering Sciences, 472 (2195), 2016. DOI:https://doi.org/10.1098/rspa.2016.0577.

[8] Yükseler, R. F., The Strain Energy Density of Compressible, Rubber-Like Shells of Revolution, ASME Journal of Applied Mechanics, 63, 419 - 423, 1996.

[9] Yükseler, R. F., On the Definition of the Deformed Reference Surface of Rubber-Like Shells of Revolution, ASME Journal of Applied Mechanics, 63, 424 - 428, 1996.

[10] Yükseler, R. F., A Theory for Rubber-Like Rods, International Journal of Solids and Structures, 69-70, 350-359, 2015.

[11] Yükseler, R. F., Local and Nonlocal Buckling of Mooney-Rivlin Rods, European Journal of Mechanics - A/ Solids, Volume 78, November-December 2019, ( https://doi.org/10.1016/j.euromechsol.2019.103816)

[12] Moon, J., Yoon, K. Y., Lee, T. H., et al. In-plane Elastic Buckling of Pin-ended Shallow Parabolic Arches. Engineering Structures, 29:2611-2617, 2017. 
[13] Altekin, M. and Yükseler, R. F., A Parametric Study on Geometrically Nonlinear Analysis of Initially Imperfect Shallow Spherical Shells, Journal of Elastomers and Plastics, 40. DOI: 10.1177/0095244307084907, 2008.

[14] Yıldırım, B. and Yükseler, R. F., Effect of Compressibility on Nonlinear Buckling of Simply Supported Polyurethane Spherical Shells Subjected to an Apical Load, Journal Of Elastomers And Plastics, 43. DOI: 10.1177/0095244310393930, 2011.

[15] Pi, Y. L. and Bradford, M. A., Non-linear Buckling and postbuckling Analysis of Arches with unequal Rotational End Restraints under a Central Concentrated Load. International Journal of Solids and Structures, 49:3762-3773, 2012.

[16] Chen, X. and Meguid, S. A., On the Parameters Which Govern the Symmetric Snapthrough Buckling Behavior of an Initially Curved Microbeam. International Journal of Solids and Structures, 66:77-87, 2015.

[17] Plaut, R. H., Snap-through of Shallow Reticulated Domes under Unilateral Displacement Control. International Journal of Solids and Structures, 148-149:24-34, 2018.

[18] Tsiatas, G. C. and Babouskos, N. G., Linear and Geometrically Nonlinear Analysis of Non-uniform Shallow Arches under a Central Concentrated Force. International Journal of Non-Linear Mechanics, 92:92-101, 2017.

[19] Virgin, L. N., Wiebe, R., Spottswood, S. M., et al. Sensitivity in the Structural Behavior of Shallow Arches. International Journal of Non-Linear Mechanics, 58:212-221, 2014.

[20] Ma, H., Fan, F., Wen, P., et al. Experimental and Numerical Studies on a Single-layer Cylindrical Reticulated Shell with Semi-rigid Joints. Thin-Walled Structures, 86:1-9, 2015.

[21] Cui, Z., Bhattacharyya, D., Moltschaniwskyj, G., Experimental and Numerical Study of Buckling Response of Composite Shells Compressed Transversely Between Rigid Platens. Composites: Part B., 36:478-486, 2005.

[22] Kolodziej, S. and Marcinowski, J., Experimental and Numerical Analyses of the Buckling of Steel, Pressurized, Spherical Shells. Advances in Structural Engineering, 21(16):2416-2432, 2018.

[23] Made, A. M., Mirmiran, A. and Nelsen, D., Stability Tests of Sandwich Composite Elastica Arches. Journal Of Structural Engineerıng, 128(5):683-686, 2002.

[24] Erdölen, A. and Yükseler, R. F., An Approach for Finite Strains and Rotations of Shells of Revolution with Application to a Spherical Shell under a Uniformly Distributed Pressure. Journal of Elastomers and Plastics, 35 (4), 357-365, October 2003, DOI:10.1177/009524403038015.

[25] Altekin, M. and Yükseler, R. F., Geometrically Nonlinear Analysis of Clamped Shallow Spherical Shells, 8th Int. Congress on Advances in Civil Engineering, Eastern, Mediterranean University, Famagusta, North Cyprus, 2008. 
[26] Yıldırım, B. and Yükseler, R. F., Combined Effect of Compressibility, Height and Thickness on the Nonlinear Behaviour of Polyurethane, Simply-Supported Spherical Shells under Apical Loads, Bitlis Eren Univ J Sci \& Technol, 4 (1), 12-19, 2014.

[27] Koçak, A. and Yükseler, R. F., Finite Axisymmetric Strains and Rotations of Shells of Revolution with Application to the Problem of a Spherical Shell under a Point Load. Sixth Annual International Conference on Composites Engineering, Orlando, Florida, 1999.

[28] Altekin, M. and Yükseler, R. F., Geometrically Nonlinear Axisymmetric Buckling Analysis of Shallow Spherical Shells. International Civil Engineering \& Arcitecture Symposium for Academicians, Side-Antalya, Turkey, 2014.

[29] Evan-Iwanowski, R. M., Loo, T. C. and Tierney, D. W., Local Buckling of Shells. Developments in Mechanics, 2:221-251, 1963.

[30] Cagan, J. and Taber, L. A., Large Deflection Stability of Spherical Shells with Ring Loads. J. Appl. Mech., 53:897-901, 1986.

[31] Ranjan, G. V., Edge Zone Expansions for Thin Shells with Application to (1) Torispherical Pressure Vessel and (2) Large Deflection of Sphere with Point Load, PhD Thesis, Stanford University, USA, 1976.

[32] Parnell, T. K., Numerical Improvement of Asymptotic Solutions and Nonlinear Shell Analysis. PhD Thesis, Stanford University, USA, 1984.

[33] Huang, N., Unsymmetrical Buckling of Thin Shallow Spherical Shells, J. Appl. Mech., 31, 447-457, 1964.

[34] Memon, B. A. and Su, X. Z., Arc-Length Technique for Nonlinear Finite Element Analysis. Journal of Zhejiang University, 5(5), 618-628, 2004.

[35] Riks, E., The Application of Newton's Method to the Problem of Elastic Stability. Journal of Applied Mechanics, 39, 1060-1065, 1972.

[36] Riks, E., An Incremental Approach to the Solution of Snapping and Buckling Problems. International Journal of Solids and Structures, 15(7), 529-551, 1979.

[37] Wempner, G. A., Discrete Approximation Related to Nonlinear Theories of Solids. International Journal of Solids and Structures, 7(11), 1581-1599, 1971.

[38] Polat, C., and Calayır Y., Lineer Olmayan Yapı Sistemlerinin Analizi İçin Yay-Boyu Metodu. FÜ Fen ve Mühendislik Bilimleri Dergisi 19.4, 525-530, 2007.

[39] Polat, C., Co-rotational F ormulation of a Solid-shell Element U tilizing the ANS and EAS Methods. Journal of Theoretical and Applied Mechanics 48.3, 771-788, 2010 . 